$\begin{array}{ll}\text { September } 1996 & 3 / 96\end{array}$

\title{
W-Strings on Curved Backgrounds
}

\author{
Aleksandar Miković đand Branislav Sazdović \& \\ Institute of Physics, P.O.Box 57, Belgrade 11001, Yugoslavia
}

\begin{abstract}
We discuss a canonical formalism method for constructing actions describing propagation of $\mathrm{W}$-strings on curved backgrounds. The method is based on the construction of a representation of the $\mathrm{W}$-algebra in terms of currents made from the string coordinates and the canonically conjugate momenta. We construct such a representation for a $W_{3}$-string propagating in the background metric with one flat direction by using a simple ansatz for the $\mathrm{W}$-generators where each generator is a polynomial of the canonical currents and the veilbeins. In the case of a general background we show that the simple polynomial ansatz fails, and terms containing the veilbein derivatives must be added.
\end{abstract}

\footnotetext{
${ }^{1}$ E-mail address: mikovic@castor.phy.bg.ac.yu

${ }^{2}$ E-mail:sazdovic@castor.phy.bg.ac.yu
} 
W-string theories are higher spin generalizations of ordinary string theories, such that the string coordinates are not only coupled to the world-sheet metric but also to a set of higher spin world-sheet gauge fields (for a review see [1]). Since ordinary string theory can be considered as a gauge theory based on the Virasoro algebra, one can analogously define a $\mathrm{W}$-string theory as a gauge theory based on a W-algebra [2] (or any other higher spin conformally extended algebra [1]). Actions for a large class of W-string theories have been constructed so far [3-10]. These actions essentially describe a W-string propagating on a flat background spacetime metric. In the case of a curved background metric, the problem of constructing invariant actions was first considered in [11], where it was solved for a special case of a group manifold. This construction was based on the canonical formalism method introduced in ref. [9]. In [11] it was crucial that a representation of the $\mathrm{W}$ algebra was known in terms of the currents which obeyed a current algebra associated with the Lie group in question. However, when the background metric was not a group manifold metric, the corresponding canonical currents did not satisfy a current algebra under the Poisson brackets. Consequently one could not obtain a representation for the $\mathrm{W}$ algebra in terms of the string coordinates and the canonicaly conjugate momenta and an invariant action could not be constructed.

In this letter we examine this problem, and show that it is caused by the ansatz used for constructing the representation of the $\mathrm{W}$ generators. The ansatz used in [11] is a simple polynomial ansatz where the $W$ generators are polynomials of the canonical currents and the veilbeins. We show that this simple polynomial ansatz also works for an arbitrary background metric with one flat direction, while in the most general case it fails. In conclusions we argue that the simple polynomial ansatz must be generalized by adding the terms containing the veilbein derivatives.

We are going to use the canonical formalism for constructing the gauge invariant actions [9]. This method works if one knows a representation of the algebra of gauge symmetries in terms of the coordinates and canonically conjugate momenta. The basic idea is simple: given a set of canonical pairs $\left(p_{i}, q^{i}\right)$ together with the Hamiltonian $H_{0}(p, q)$ and the constraints $G_{\alpha}(p, q)$ such that

$$
\begin{aligned}
& \left\{G_{\alpha}, G_{\beta}\right\}=f_{\alpha \beta}^{\gamma} G_{\gamma}, \\
& \left\{G_{\alpha}, H_{0}\right\}=h_{\alpha}^{\beta} G_{\beta}
\end{aligned}
$$

where $\{$,$\} is the Poisson bracket and (1) is the desired algebra of gauge symmetries,$ then the corresponding action is given by

$$
S=\int d t\left(p_{i} \dot{q}^{i}-H_{0}-\lambda^{\alpha} G_{\alpha}\right)
$$


The parameter $t$ is the time and dot denotes time derivative. The Lagrange multipliers $\lambda^{\alpha}(t)$ play the role of the gauge fields associated with the gauge symmetries generated by $G_{\alpha}$. The indices $i, \alpha$ can take the discrete as well as the continious values. Note that the coefficients $f_{\alpha \beta}{ }^{\gamma}$ and $h_{\alpha}{ }^{\beta}$ can be arbitrary functions of $p_{i}$ and $q^{i}$, and hence the algebra (1) is general enough to accomodate the case of the $W$ algebras, where the right-hand side of the Eq. (1) is a non-linear function of the generators. The action $S$ is invariant under the following gauge transformations

$$
\begin{aligned}
\delta p_{i} & =\epsilon^{\alpha}\left\{G_{\alpha}, p_{i}\right\} \\
\delta q^{i} & =\epsilon^{\alpha}\left\{G_{\alpha}, q^{i}\right\} \\
\delta \lambda^{\alpha} & =\dot{\epsilon}^{\alpha}-\lambda^{\beta} \epsilon^{\gamma} f_{\gamma \beta}{ }^{\alpha}-\epsilon^{\beta} h_{\beta}{ }^{\alpha} .
\end{aligned}
$$

It can be seen from the the transformation law for $\lambda^{\alpha}$ why they can be identified as gauge fields.

Since we want to describe propagation of a bosonic W-string on a curved background, the canonical coordinates will be a set of two-dimensional $(2 \mathrm{~d})$ scalar fields $\phi^{A}(\sigma, \tau), A=1, \ldots, N$, where $\sigma$ is the string coordinate $(0 \leq \sigma \leq 2 \pi)$ and $\tau$ is the evolution parameter. $\phi^{A}$ will be coordinates on an $N$-dimensional space-time manifold $M$. On $M$ is also given a metric $G_{A B}$, which can be of arbitrary signature. Let $\pi_{A}(\sigma, \tau)$ be the canonically conjugate momenta, satisfying

$$
\left\{\phi^{A}\left(\sigma_{1}, \tau\right), \pi_{B}\left(\sigma_{2}, \tau\right)\right\}=\delta_{B}^{A} \delta\left(\sigma_{1}-\sigma_{2}\right)
$$

In order to construct an invariant action, we will need a canonical representation of the corresponding $\mathrm{W}$-algebra. We start from the action for an ordinary bosonic string propagating on $M$, which is a $2 \mathrm{~d} \sigma$-model action

$$
S_{2}=\int d^{2} \sigma \frac{1}{2} \sqrt{-g} g^{\mu \nu} \partial_{\mu} \phi^{A} \partial_{\nu} \phi^{B} G_{A B}(\phi)
$$

The action (6) can be rewritten in a canonical form as

$$
S_{2}=\int_{\tau_{1}}^{\tau_{2}} d \tau \int_{0}^{2 \pi} d \sigma\left(\pi_{A} \dot{\phi}^{A}-h^{\alpha} T_{\alpha}\right)
$$

where $\alpha=\{+,-\}$ and the constraints $T_{\alpha}$ are given by

$$
T_{ \pm}=\frac{1}{2} G^{A B} J_{ \pm A} J_{ \pm B}
$$

We have introduced the currents

$$
J_{ \pm A}=\frac{1}{\sqrt{2}}\left(\pi_{A} \pm G_{A B} \phi^{\prime B}\right)
$$


and the primes stand for the $\sigma$ derivatives. The constraints $T_{\alpha}$ are ++ and -components of the string energy-momentum tensor $T_{\mu \nu}$, where $x^{ \pm}=x^{0} \pm x^{1}$. $T_{\alpha}$ satisfy the classical Virasoro algebra

$$
\left\{T_{ \pm}\left(\sigma_{1}\right), T_{ \pm}\left(\sigma_{2}\right)\right\}= \pm \delta^{\prime}\left(\sigma_{1}-\sigma_{2}\right)\left(T_{ \pm}\left(\sigma_{1}\right)+T_{ \pm}\left(\sigma_{2}\right)\right)
$$

under the Poisson brackets (5). The Poisson brackets of the currents $J_{\alpha A}$ are given by

$$
\begin{aligned}
\left\{J_{ \pm A}\left(\sigma_{1}\right), J_{ \pm B}\left(\sigma_{2}\right)\right\}= & \pm \frac{1}{2}\left(\partial_{B} G_{A C}-\partial_{A} G_{B C}\right) \phi^{\prime C} \delta\left(\sigma_{1}-\sigma_{2}\right) \\
& \pm \frac{1}{2}\left[G_{A B}\left(\sigma_{1}\right)+G_{A B}\left(\sigma_{2}\right)\right] \delta^{\prime}\left(\sigma_{1}-\sigma_{2}\right)
\end{aligned}
$$

For a general metric $G_{A B}$ the relations (11) do not form a current algebra, and the currents of opposite chirality do not have vanishing Possion brackets, but

$$
\begin{aligned}
\left\{J_{+A}\left(\sigma_{1}\right), J_{-B}\left(\sigma_{2}\right)\right\} & =\phi^{\prime C} \Gamma_{C, A B} \delta\left(\sigma_{1}-\sigma_{2}\right) \\
& \equiv \phi^{\prime C} \frac{1}{2}\left(\partial_{A} G_{B C}+\partial_{B} G_{A C}-\partial_{C} G_{A B}\right) \delta\left(\sigma_{1}-\sigma_{2}\right),
\end{aligned}
$$

where $\Gamma_{C, A B}$ is the Christoffel symbol. When $G_{A B}$ is a group manifold metric, then the relations (11) and (12) can give two independent chiral current algebras [11].

Although the currents (9) do not form a closed current algebra, we will proceed with the ansatz from the group metric case in order to find its limitations. We take

$$
W_{\alpha s}=\frac{1}{s} D^{A_{1} \cdots A_{s}} J_{\alpha A_{1}} \cdots J_{\alpha A_{s}} \quad(s=2, \ldots, N),
$$

where the coefficients $D^{A_{1} \ldots A_{s}}$ will be determined from the requirement that the Poisson brackets of the quantities (13) form a $W$ algebra. Note that from (8) we have $D^{A B}=G^{A B}$. For the sake of simplicity we specialize to the $W_{3}$-string case. The expressions (13) should then obey a classical $W_{3}$ algebra $\left(W \equiv W_{3}\right)$

$$
\begin{gathered}
\left\{T_{ \pm}\left(\sigma_{1}\right), T_{ \pm}\left(\sigma_{2}\right)\right\}= \pm \delta^{\prime}\left(\sigma_{1}-\sigma_{2}\right)\left(T_{ \pm}\left(\sigma_{1}\right)+T_{ \pm}\left(\sigma_{2}\right)\right) \\
\left\{T_{ \pm}\left(\sigma_{1}\right), W_{ \pm}\left(\sigma_{2}\right)\right\}= \pm \delta^{\prime}\left(\sigma_{1}-\sigma_{2}\right)\left(W_{ \pm}\left(\sigma_{1}\right)+2 W_{ \pm}\left(\sigma_{2}\right)\right) \\
\left\{W_{ \pm}\left(\sigma_{1}\right), W_{ \pm}\left(\sigma_{2}\right)\right\}= \pm \delta^{\prime}\left(\sigma_{1}-\sigma_{2}\right)\left(T^{2}{ }_{ \pm}\left(\sigma_{1}\right)+T^{2}{ }_{ \pm}\left(\sigma_{2}\right)\right)
\end{gathered}
$$

From the relation (14b) it follows that $D^{A B C}$ is a covariantly constant tensor

$$
\nabla_{D} D^{A B C}=\partial_{D} D^{A B C}+\Gamma_{D E}^{(A} D^{B C) E}=0
$$

while from the relation $(14 \mathrm{c})$ it follows that

$$
D_{E}^{(A B} D^{C D) E}=\frac{1}{2} G^{(A B} G^{C D)} .
$$


If we introduce the veilbeins $E_{a}^{A}(\phi)$ as

$$
G^{A B}=\eta^{a b} E_{a}^{A} E_{b}^{B}
$$

where $\eta_{a b}$ is a flat metric, then the equation (15) is satisfied if

$$
D^{A B C}=\Delta^{a b c} E_{a}^{A} E_{b}^{B} E_{c}^{C}
$$

where $\Delta^{a b c}$ are $\phi$-independent coefficients and

$$
\nabla_{A} E_{b}^{B}=\partial_{A} E_{b}^{B}+\Gamma_{A C}^{B} E_{b}^{C}=0
$$

Then the condition (16) becomes

$$
\Delta_{e}^{(a b} \Delta^{c d) e}=\frac{1}{2} \delta^{(a b} \delta^{c d)}
$$

which is satisfied for $\Delta^{a b c}=d^{a b c}$, where $d^{a b c}$ are the flat-background coefficients [9]. However, there are further conditions which $D^{A B C}$ have to satisfy, and they come from the requiriments

$$
\left\{T_{+}, W_{-}\right\}=0 \quad, \quad\left\{T_{-}, W_{+}\right\}=0 \quad, \quad\left\{W_{+}, W_{-}\right\}=0
$$

Equations (21) give the following constraints

$$
\begin{aligned}
0 & =-\frac{1}{3} G^{A B} \partial_{B} D^{C D E} J_{ \pm A} J_{\mp C}+\frac{1}{2} \partial_{C} G^{A B} D^{C D E} J_{ \pm A} J_{ \pm B} \pm \sqrt{2} G^{A B} D^{C D E} \Gamma_{F, A C} J_{ \pm B} \phi^{\prime F} \\
0 & =-\frac{1}{3} D^{A B C} \partial_{C} D^{D E F} J_{+A} J_{+B} J_{-D}+\frac{1}{3} \partial_{D} D^{A B C} D^{D E F} J_{+A} J_{+B} J_{+C} \\
& +\sqrt{2} D^{A B C} D^{D E F} \Gamma_{H, C D} J_{+A} J_{+B} \phi^{\prime H}
\end{aligned}
$$

By using (9), the constraints (22) can be rewritten as polynomials in $\pi_{A}$ and $\phi^{\prime A}$, and (22) will be satisfied if the coeficients of these polynomials vanish. In this way one obtains the additional constraints on the solution (18). Hence it is clear that (18) is a solution only for a special class of backgrounds, and one example is the group manifold case [11]. A second example will be constructed here, and it is given by a background metric with one flat direction. Note that if one wants to find new solutions, the strategy of solving the constraints (15) (16) and (22) is not very efficient, and better thing to do is to look among existing representations of $W_{3}$ in terms of scalar fields.

In order to construct the solution for the background with one flat direction, we start from a flat-background representation

$$
T_{ \pm}=\frac{1}{2} \eta^{a b} J_{ \pm a} J_{ \pm b} \quad, \quad W=\frac{1}{3} d^{a b c} J_{ \pm a} J_{ \pm b} J_{ \pm c}
$$


where the non-zero $d^{a b c}$ are given by

$$
d^{111}=\frac{1}{\sqrt{2}} \quad, \quad d^{1 i j}=-\frac{\eta^{i j}}{\sqrt{2}} \quad, \quad(i=2, \ldots, N)
$$

Equation (23) can be then rewritten as

$$
T_{ \pm}=\frac{1}{2} \eta^{11} J_{ \pm 1}^{2}+T_{ \pm 2} \quad, \quad W_{ \pm}=\frac{1}{3 \sqrt{2}} J_{ \pm 1}^{3}-\sqrt{2} J_{ \pm 1} T_{ \pm 2}
$$

where $T_{ \pm 2}=\frac{1}{2} \eta^{i j} J_{ \pm i} J_{ \pm j}$ is the energy-momentum tensor of the fields $\phi^{i}$. Note that the representation (25) is valid even when $T_{2}$ is an arbitrary $2 \mathrm{~d}$ energy-momentum tensor as long as $\left\{J_{1}, T_{2}\right\}=0$. This property allows us to construct a $W_{3}$ representation for a curved background with one flat direction

$$
G_{11}= \pm 1 \quad, \quad G_{1 i}=0 \quad, \quad G_{i j}=g_{i j} \quad, \quad \partial_{1} g_{i j}=0
$$

where $g_{i j}$ is an arbitrary $(N-1)$-dimensional metric. Namely, $T_{2}$ in that case follows from the formulas (8) and (9), while $J_{1}$ is given by (9). The corresponding representation is given by the formula (25), and it is of the form (18), where now $E_{a}^{A}$ are associated to the metric (26).

The $W_{3}$-string action in the background (26) now follows from the general formula (3)

$$
S_{3}=\int_{\tau_{1}}^{\tau_{2}} d \tau \int_{0}^{2 \pi} d \sigma\left(\pi_{A} \dot{\phi}^{A}-\sum_{s=2}^{3} b_{s}{ }^{\alpha} W_{\alpha s}\right)
$$

where

$$
T_{ \pm}=\frac{1}{2} G^{11} J_{ \pm 1}^{2}+\frac{1}{2} g^{i j} J_{ \pm i} J_{ \pm j} \quad, \quad W_{ \pm}=\frac{1}{3 \sqrt{2}} J_{ \pm 1}^{3}-\frac{1}{\sqrt{2}} g^{i j} J_{ \pm 1} J_{ \pm i} J_{ \pm j}
$$

As discussed in [9] the $2 \mathrm{~d}$ diffeomorphism invariance requires $H_{0}=0$, while $b_{s}^{\alpha}$ are the lagrange multipliers, which are also the gauge fields corresponding to the $W$ symmetries. The gauge transformation laws can be determined from the Eq. (4), and in the $W_{3}$ case we obtain

$$
\begin{aligned}
\delta \pi_{A} & =\frac{1}{2}\left\{\epsilon^{\alpha} \partial_{A} G^{B C} J_{\alpha B} J_{-\alpha C}+\xi^{\alpha} D^{B C D}\left[\partial_{A} G_{B E} J_{-\alpha}^{E}+\left(\partial_{B} G_{E A}-\partial_{E} G_{B A}\right) J_{\alpha}^{E}\right] J_{\alpha C} J_{\alpha D}\right\} \\
& -\frac{(-)^{\alpha}}{\sqrt{2}}\left(\epsilon^{\alpha} J_{\alpha A}+\xi^{\alpha} D_{A}^{B C} J_{\alpha B} J_{\alpha C}\right)^{\prime} \\
\delta \phi^{A} & =-\frac{1}{\sqrt{2}}\left(\epsilon^{\alpha} J_{\alpha}{ }^{A}+\xi^{\alpha} D^{A B C} J_{\alpha B} J_{\alpha C}\right) \\
\delta h^{\alpha} & =\dot{\epsilon}^{\alpha}-(-1)^{\alpha}\left[h^{\alpha}\left(\epsilon^{\alpha}\right)^{\prime}-\left(h^{\alpha}\right)^{\prime} \epsilon^{\alpha}\right]+(-1)^{\alpha}\left[\xi^{\alpha}\left(b^{\alpha}\right)^{\prime}-\left(\xi^{\alpha}\right)^{\prime} b^{\alpha}\right] T_{\alpha} \\
\delta b^{\alpha} & =\dot{\xi}^{\alpha}+(-1)^{\alpha}\left[2\left(h^{\alpha}\right)^{\prime} \xi^{\alpha}-h^{\alpha}\left(\xi^{\alpha}\right)^{\prime}-2 b^{\alpha}\left(\epsilon^{\alpha}\right)^{\prime}+\left(b^{\alpha}\right)^{\prime} \epsilon^{\alpha}\right]
\end{aligned}
$$


where $h^{\alpha}=b_{2}^{\alpha}, b^{\alpha}=b_{3}^{\alpha}, \epsilon^{ \pm}$are the parameters of the $T_{ \pm}$transformations, while $\xi^{ \pm}$ are the parameters of the $W_{ \pm}$transformations. In all equations we use Einstein's summation convention, i.e. summation is performed only if the up and down index are the same.

In order to find a geometrical interpretation of the action (27) we need to know its second order form. It can be obtained by replacing the momenta $\pi_{A}$ in (27) by their expressions in terms of $\phi^{A}$. These expressions can be obtained from the equation of motion

$$
\frac{\delta S_{3}}{\delta \pi_{A}}=\dot{\phi}^{A}-\frac{h^{\alpha}}{\sqrt{2}} J_{\alpha}^{A}-\frac{1}{\sqrt{2}} b^{\alpha} D^{A B C} J_{\alpha B} J_{\alpha C}=0
$$

This is a quadratic equation in $\pi_{A}$, and therefore the second order form of the Lagrangean density of (27) will be a non-polynomial function of $\partial_{\mu} \phi^{A}, h^{\alpha}$ and $b^{\alpha}$. Since every solution of (30) can be written as an infinite power series in $\partial_{\mu} \phi^{A}$, the Lagrangean density will also be an infinite power series in $\partial_{\mu} \phi^{A}$, in a complete analogy with the flat background case [9] and the group manifold case [11].

In the $W_{2}$ case one can show that after the elimination of the momenta in (7) one obtains the covariant action (6), after the following identifications

$$
\tilde{g}^{00}=\frac{2}{h^{+}+h^{-}} \quad, \quad \tilde{g}^{01}=\frac{h^{-}-h^{+}}{h^{+}+h^{-}} \quad, \quad \tilde{g}^{11}=-\frac{2 h^{+} h^{-}}{h^{+}+h^{-}},
$$

where $\tilde{g}^{\mu \nu}=\sqrt{-g} g^{\mu \nu}$. The covariant form of the $2 \mathrm{~d}$ diffeomorphism transformations can be obtained from the Eq. (29.b), by rewritting it as

$$
\delta \phi^{A}=-\frac{\epsilon^{\alpha}}{\sqrt{2}} J_{\alpha}{ }^{A}=-\frac{\epsilon^{\alpha}}{\sqrt{h^{+}+h^{-}}} \tilde{e}_{\alpha}{ }^{\mu} \partial_{\mu} \phi^{a}=\epsilon^{\mu} \partial_{\mu} \phi^{a}
$$

where

$$
\tilde{e}_{\alpha}^{\mu}=\frac{1}{\sqrt{h^{+}+h^{-}}}\left(\begin{array}{cc}
1 & h^{-} \\
1 & -h^{+}
\end{array}\right) .
$$

Eq. (29.c) can be rewritten as

$$
\delta \tilde{g}^{\mu \nu}=-\partial_{\rho}\left(\epsilon^{\rho} \tilde{g}^{\mu \nu}\right)+\partial_{\rho} \epsilon^{(\mu \mid} \tilde{g}^{\mid \nu) \rho},
$$

which is the diffeomorphism transformation of a densitized metric generated by the parameter $\epsilon^{\mu}$. The metric $g^{\mu \nu}$ can be written as

$$
g^{\mu \nu}=\frac{1}{\sqrt{-g}\left(h^{+}+h^{-}\right)}\left(\begin{array}{cc}
2 & h^{-}-h^{+} \\
h^{-}-h^{+} & -2 h^{+} h^{-}
\end{array}\right)=e_{+}{ }^{(\mu \mid} e_{-}{ }^{\mid \nu)},
$$

where $e_{\alpha}{ }^{\mu}=(-g)^{-\frac{1}{4}} \tilde{e}_{\alpha}{ }^{\mu}$ are the zweibeins. Note that $\sqrt{-g}$ remains undetermined, because the action (6) is independent of $\sqrt{-g}$ due to the Weyl symmetry

$$
\delta g^{\mu \nu}=\omega g^{\mu \nu}
$$


Also note that the relations (31),(33) and (35) are essentialy the same as in the flat background case [9], as well as in the group manifold case [11].

In the $W_{3}$ case we have from the Eq. (30)

$$
\begin{aligned}
\pi_{A} & =G_{A B} \tilde{g}^{0 \mu} \partial_{\mu} \phi^{B}+\sqrt{2 \tilde{g}^{00}} \delta \Pi_{A} \\
\delta \Pi_{A} & =-\frac{\sqrt{\tilde{g}^{00}} b^{\alpha}}{2} D_{A}^{B C} J_{\alpha B} J_{\alpha C} \equiv-\frac{1}{2} B^{\alpha} D_{A}{ }^{B C} \bar{J}_{\alpha B} \bar{J}_{\alpha C},
\end{aligned}
$$

where

$$
\bar{J}_{\alpha}^{A}=\left(\tilde{g}^{00}\right)^{-\frac{1}{2}} J_{\alpha}^{A} \quad, \quad B^{\alpha}=\left(\tilde{g}^{00}\right)^{\frac{3}{2}} b^{\alpha},
$$

and $\tilde{g}^{00}$ is given by the Eq. (31). Then the action (27) takes the following form

$$
S_{3}=\int d^{2} \sigma\left(\mathcal{L}_{2}-\delta \Pi^{A} \delta \Pi_{A}-\frac{B^{\alpha}}{3} D^{A B C} \bar{J}_{\alpha A} \bar{J}_{\alpha B} \bar{J}_{\alpha C}\right)
$$

where $\mathcal{L}_{2}$ is the Lagrangean density of the action (6). Note that the action (39) can be written in a more elegant form

$$
S_{3}=\int d^{2} \sigma\left(\bar{J}_{+A} \partial_{-} \phi^{A}+\bar{J}_{-A} \partial_{+} \phi^{A}-\bar{J}_{+}^{A} \bar{J}_{-A}-\frac{B^{\alpha}}{3} D^{A B C} \bar{J}_{\alpha A} \bar{J}_{\alpha B} \bar{J}_{\alpha C}\right),
$$

where we have used the equation (30) rewritten as

$$
\bar{J}_{ \pm}^{A}=\partial_{ \pm} \phi^{A}-\frac{1}{2} B^{\alpha} D^{A B C} \bar{J}_{\alpha B} \bar{J}_{\alpha C}
$$

Eq. (41) can be used to obtain a power series expansion of $\bar{J}_{A}$ in terms of $\partial_{ \pm} \phi, h$ and $B$, which can be inserted into Eq. (40) to give the corresponding power series expansion of the action. Up to the second order in $B$ the Lagrangean desity can be written as

$$
\begin{aligned}
\mathcal{L}_{3} & =\mathcal{L}_{2}-\frac{1}{3} B^{\alpha} D_{A B C} \partial_{\alpha} \phi^{A} \partial_{\alpha} \phi^{B} \partial_{\alpha} \phi^{C} \\
& +\frac{1}{4} B^{\alpha} B^{\beta} D_{A B C} D^{A}{ }_{D E} \partial_{\alpha} \phi^{B} \partial_{\alpha} \phi^{C} \partial_{\beta} \phi^{D} \partial_{\beta} \phi^{E}+O\left(B^{3}\right)
\end{aligned}
$$

It is clear that the covariant form of (42) will be

$$
\begin{aligned}
\mathcal{L}_{3}= & \frac{1}{2} \tilde{g}^{\mu \nu} G^{A B}(\phi) \partial_{\mu} \phi^{A} \partial_{\nu} \phi^{B}+\frac{1}{3} \tilde{b}^{\mu \nu \rho} D_{A B C}(\phi) \partial_{\mu} \phi^{A} \partial_{\nu} \phi^{B} \partial_{\rho} \phi^{C} \\
& +\frac{1}{4} \tilde{c}^{\mu \nu \rho \sigma} D_{A B}^{E}(\phi) D_{E C D}(\phi) \partial_{\mu} \phi^{A} \partial_{\nu} \phi^{B} \partial_{\rho} \phi^{C} \partial_{\sigma} \phi^{D}+\cdots
\end{aligned}
$$

where the objects $\tilde{g}, \tilde{b}, \tilde{c}, \ldots$, must transform as $2 \mathrm{~d}$ tensor densities since the action is invariant under the infinitesimal diffeomorphisms

$$
\delta \phi^{A}=-\frac{\epsilon^{A}}{\sqrt{2}} J_{\alpha}^{A}=\epsilon^{\mu} \partial_{\mu} \phi^{A} \quad, \quad \epsilon^{\mu}=f^{\mu}\left(\epsilon^{ \pm}, h^{ \pm}, b^{ \pm}, \phi^{A}, \partial_{\mu} \phi^{A}\right)
$$


The action (43) is also invariant under a generalized Weyl symmetry [1]. This symmetry is built in by our construction, since we used only four independent gauge fields $h^{ \pm}$and $B^{ \pm}$. Consequently, the fields $\tilde{g}, \tilde{b}, \tilde{c}, \ldots$ are functions of $h$ and $b$, and one can check order by order in $\partial \phi$ that

$$
\tilde{g}_{\mu \nu} \tilde{b}^{\mu \nu \rho}=0 \quad, \quad \tilde{c}^{\mu \nu \rho \sigma}=\tilde{g}_{\tau \epsilon} \tilde{b}^{\mu \nu \tau} \tilde{b}^{\epsilon \rho \sigma}
$$

and so on. Therefore the independent gauge fields are the $2 \mathrm{~d}$ metric $g_{\mu \nu}$ and a symmetric tensor $b_{\mu \nu \rho}$, related to $\tilde{g}$ and $\tilde{b}$ as

$$
\tilde{g}_{\mu \nu}=\sqrt{-g} g_{\mu \nu} \quad, \quad \tilde{b}_{\mu \nu \rho}=\sqrt{-g}\left(b_{\mu \nu \rho}-\frac{3}{2} g_{(\mu \nu \mid} b_{\sigma \mid \rho)}^{\sigma}\right)
$$

In conclusion we can say that the second order Lagrangean (43) for the $W_{3}$-string propagating in the background metric (26) has the same form as in the group-manifold case [11]. The appearance of the same generic form for the action in both cases may lead one to belive that (43) is valid for a general case. However, it is clear from our analysis that the relation (18) cannnot be satisfied for a general background metric. In that case one can try to generalize Eq. (18) by allowing $\Delta^{a b c}$ to become functions of $\phi^{A}$. Since $\Delta^{a b c}$ must be built out of $\eta_{a b}, d^{a b c}$ and the veilbeins, the only way to make $\Delta^{a b c} \phi$-dependent is to allow the veilbein derivatives to appear in the expressions for $\Delta^{a b c}$. For example

$$
\Delta^{a b c}=d^{a b c}+c_{1} \partial_{A} E^{A a} \partial_{B} E^{B b} \partial_{C} E^{C c}+\ldots,
$$

where $c_{1}$ is a numerical constant. Given the fact that $D^{A B C}$ has to be highly constrained, it is difficult to see whether the modification (47) can yield a solution in terms of the unconstrained vielbeins. A more likely possibility is to change the original ansatz for the $\mathrm{W}$ generators (13), by adding the terms in $W_{s}$ which contain higher powers of $J$ then the conformal spin $s$. The coefficients of these terms must be proportional to the vielbein derivatives, since they vanish in the flat case. A more precise functional dependence can be obtained from the fact that these terms also vanish in the group-manifold case and in the case of the metric (26). In the lowest order, the new ansatz can be written as

$$
W_{\alpha 3}=\frac{1}{3} D^{A B C} J_{\alpha A} J_{\alpha B} J_{\alpha C}+F_{\alpha}^{A B C D} J_{\alpha A} J_{\alpha B} J_{\alpha C} J_{\alpha D}+\ldots
$$

where $F_{\alpha}$ is built from $\eta_{a b}, d_{a b c}$, veilbeins and the derivatives of veilbeins. For example $\nabla^{(A} D^{B C D)}$ would be a consistent term in $F^{A B C D}$. However, an exact determination of $W$ would require a separate paper, and it would be interesting to find out whether the power series in $J$ in (48) terminates after a finite number of terms and whether the terms containing the opposite chirality currents have to be added. 
Acknowledgements: We would like to thank MNTRS for a financial support and Simon Ljakhevich for usefull discussions.

\section{REFERENCES}

[1] C.M. Hull, Classical and quantum W-gravity, in Strings and Symmetries 1991, eds. N. Berkovits et al., World Scientific, Singapore (1992)

[2] A.B. Zamolodchikov, Teor. Mat. Fiz. 65 (1985) 1205

V.A. Fateev and A.B. Zamolodchikov, Nucl. Phys. B280 (1987) 644

V.A. Fateev and S. Lukyanov, Int. J. Mod. Phys. A3 (1988) 507

[3] C.M. Hull, Phys. Lett. 240B (1990) 110

[4] K. Schoutens, A. Sevrin and P. van Nieuwenhuizen, Phys. Lett. 243B (1990) 245

[5] E. Bergshoeff, C.N. Pope, L.J. Romans, E. Sezgin, X. Shen and K.S. Stelle, Phys. Lett. 243B (1990) 350

[6] C.M. Hull, Nucl. Phys. B353 (1991) 707

[7] C.M. Hull, Phys. Lett. 259B (1991) 68

[8] A. Miković, Phys. Lett. 260B (1991) 75

[9] A. Miković, Phys. Lett. 278B (1991) 51

[10] G.M. Sotkov, M. Stanishkov and C.-J. Zhu, Nucl. Phys. B356 (1991) 245, 439

[11] A. Miković and B. Sazdović, Mod. Phys. Lett. A 10 (1995) 1041 